\title{
Research on Hyperspectral Unmixing Oil Spill Monitoring
}

\author{
Can CUI \\ Navigation College. Dalian Maritime University \\ Dalian, China \\ 173330967@qq.com \\ Hong-ji CHEN \\ 61206 Troops of PLA \\ Dalian, China \\ 642080454@qq.com \\ Jin XU \\ Navigation College. Dalian Maritime University \\ Dalian, China \\ xujin_vip@126.com
}

\author{
Ying $\mathrm{LI}^{*}$ \\ Navigation College. Dalian Maritime University \\ Dalian, China \\ yldmu@126.com \\ Bing-xin LIU \\ Navigation College. Dalian Maritime University \\ Dalian, China \\ liubx733@126.com \\ Guan-nan LI \\ Navigation College. Dalian Maritime University \\ Dalian, China \\ 1010113796@qq.com
}

\begin{abstract}
Hyperspectral unmixing is an important technique for hyperspectral image analysis. In this paper, we took Airborne Visible Infrared Imaging Spectrometer (AVIRIS) hyperspectral imagery as dataset to monitor oil spills. The information of oil spills was retrieved through image preprocessing, minimum noise fraction (MNF) feature extraction, endmember extraction (pure pixel index (PPI), unsupervised orthogonal subspace projection (UOSP)) and fully constrained least squares (FCLS) abundance estimation. In the steps of endmember extraction, the experiment measured endmember spectra of oil and water were used as reference spectra. Then we compared the endmember spectra extracted in the image to the measured spectra by the spectral angle. At last the FCLS abundance estimation was carried on to evaluate the endmember extraction quality. The result demonstrates that the unsupervised OSP-FCLS model is better than supervised PPI-FCLS endmember extraction.
\end{abstract}

Keywords-remote sensing; hyperspectral unmixing; oil spill; endmember; abundance estimation

\section{INTRODUCTION}

With the rapid growth in oil consumption and transportation at sea in recent years, it has brought the oil spill accidents frequently, which are harmful to marine ecological environment. Nowadays oil spill monitoring has become the focus of current research topics. In terms of marine oil pollution monitoring and management, it needs to be accurate, quick and reliable. With the hyperspectral technology developments, application of hyperspectral remote sensing oil spill detection has received highly attention, which is good at the quantitative monitoring on oil film type, thickness, area and scale. Although hyperspectral images could provide rich spectral information, because of the lower spatial resolution, the mixed pixel phenomenon is common. The mixed pixels are an important reason to affect the classification accuracy of remote sensing images and the effect of target detection. During to the classification of remote sensing images and information of different features, it is difficult to give a specific category of mixed pixel. Different classifiers and even the different parameters of the same classifier may make a difference in the category of a mixed pixel. Therefore, it is an important content in the study of hyperspectral remote sensing to solve the mixed pixel problem. P. Sidike compared the complete spectral unmixing and partial spectral unmixing for the marine oil spill detection [1]. D. Sykas presented the fully constrained network based on unmixing method to estimate the oil spill thickness [2].

Reference spectra of oil and water are measured by ASD spectrometer in this paper, and compared with the results of spectra extracted from the hyperspectral image endmember. The PPI model and UOSP model are used to extract the endmember. Then, the abundance map is obtained through the FCLS method. Qualitative and quantitative evaluations are combined with visual analysis and root mean square error (RMSE) of the hyperspectral unmixing results, the flowchart is shown in Fig.1. 


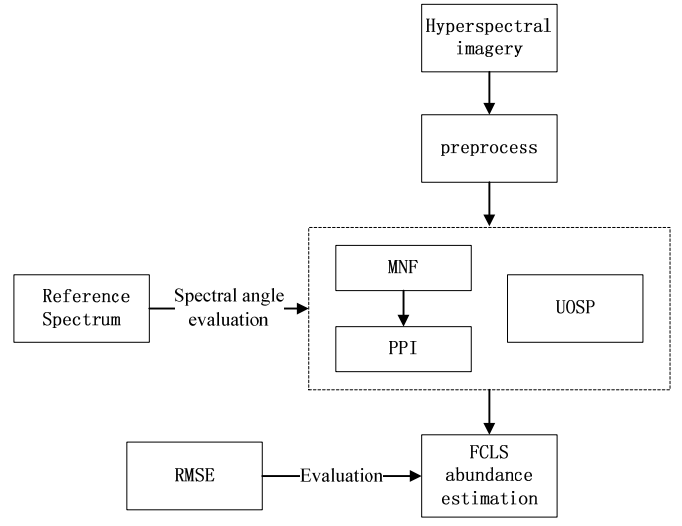

Figure 1. Flowchart

II.

\section{REFERENCE ENDMEMBER SPECTRA COLLECTION}

Crude oil (Gulf of Mexico oil type) was chosen in the experiment as the reference oil. The experiment was equiped with the United States ASD FieldSpec $® 3$ spectrometer and a drums simulating the infinite deep water environment about $60 \mathrm{~cm}$ in depth, $50 \mathrm{~cm}$ in diameter, coated with black matte paint. The measurement site was located on the roof surrounding no higher buildings and no stray light. A cloudless sky, weak wind was necessary to ensure the water was in a static state. The endmember spectra were obtained through data preprocessing (excluding abnormal curve, spectral reflectance calculation through the target and the brightness value of the reference plate). There measured reflectance spectral curves of different oil thickness. As is shown in Fig. 2, the water has the lowest value. The thinner the oil film thickness, the more like the shape of the water spectrum curve. At the wavelength of $533 \mathrm{~nm}$, there is an overall change from convex to concave. When the oil film thickness reaches $1500 \mu \mathrm{m}-2000 \mu \mathrm{m}$, there is also a sharp peak at wavelength of $783 \mathrm{~nm}$ [3]. Because there are many water vapor absorption bands in the $950 \mathrm{~nm}-2300 \mathrm{~nm}$ range and there is no obvious difference between oil and water, this range was excluded.

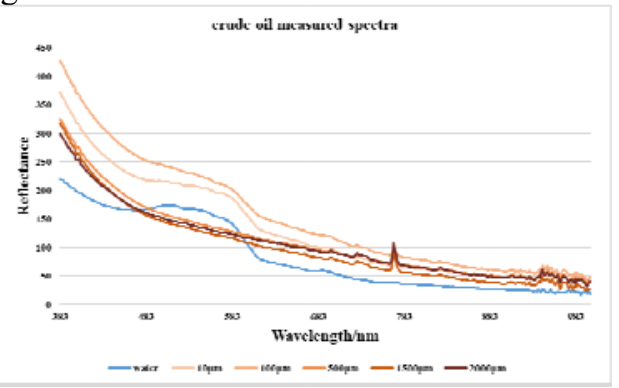

Figure 2. Measured spectra of crude oil

III.

ENDMEMBER EXTRACTION

In the present study, Airborne Visible Infrared Imaging Spectrometer (AVIRIS) hyperspectral imagery was served as the dataset, digitized in 12bit. It has a spatial resolution of $15 \mathrm{~m}$ and $224 \mathrm{bands}$. Among them, band 1-5, 107-113, 150-166, 221-224 were absorpt by water vapor and removed from the data. The remaining 158 bands were taken as data sources for spectral unmixing. A $418 * 839$ pixel AVIRIS image was acquired on 13th May, 2010, covering an area from $28^{\circ} 4^{\prime} 42.75^{\prime \prime} \mathrm{N}$ to $28^{\circ} 6^{\prime} 55.90^{\prime \prime} \mathrm{N}, 88^{\circ} 42.40^{\prime \prime} \mathrm{W}$ to $88^{\circ} 1^{\prime} 55.93^{\prime \prime} \mathrm{W}$. The image was preprocessed by atmospheric correction, spectral subset, spatial subset, and bad line repair.

Because the spatial resolution of hyperspectral image is generally low, there are many features in a pixel. Endmember is equivalent to a pixel in the subpixel, containing only spectral information of one feature. During the hyperspectral unmixing, endmember abundance can described quantitatively how much endmember in this pixel and the percentage of each endmember in this pixel.

Endmember is generally derived from two methods: first, the reference endmember, from the standard spectral library or the actual measurement of surface features; two, image endmember, from the image by the supervised or unsupervised to get the endmember of the spectrum. In this paper, we used supervised purity index pixel method and unsupervised subspace projection orthogonal method to obtain the image endmember.

\section{A. PPI}

By far, the most commonly used endmember extraction tool is PPI, which searches for vertices that define the data volume in n-dimensional feature space. PPI algorithm is based on linear spectral mixture model geometry. As the endmember is the terminal vertex of the image feature space. In the feature space, several lines randomly generated, and all pixels were projected to each line. Two points in the projection on extreme edge was the end point of the projection element. For each pixel, pure pixel index was defined which records the times being the end points of the projection. Then the larger the pure pixel index is, the higher possibility of endmember [4].

Because of the hyperspectral imagery huge band numbers, the computation is too large and the noise is high, which leads to the time consuming, the difficulty of computation, and the redundancy. Before the application of PPI endmember extraction, the feature extraction was used to reduce the dimensions of the images.

Common feature extraction methods include MNF transform and principal component transform (PCA), etc. MNF is similar to PCA in that involves two cascading PCA transformations, where the first estimates a noise covariance matrix to decorrelate and rescale the noise in the data. The next is a standard PCA of the noise-reduced data. The assumption here is that the image endmembers lie within the first few principal component axes, whereas the remaining axes are related to noise. In order to further spectral processing, the dimensions of the useful information is determined by examining the final eigenvalues and the related MNF images.

Input: $R$ denotes dimension reduction image, $\mathrm{m}$ denotes endmember number, $k_{\max }$ denotes maximum iterations.

Output: $\tilde{E}$ denotes endmember after dimension reduction.

Step 1 Define the ppi set $\left\{p_{i}\right\}_{i=1}^{n}$, here $p_{i}=0, \forall i$. 4.

Step 2 For each $k=1 \ldots \ldots, k_{\max }$, execute Step 3 and Step

Step 3 Generate unit random vector and calculate the projection of the image in the direction of $\beta_{k}, R_{k}^{\prime}=\beta_{k} \tilde{R}$. 
Step4 Search for the location of the maximum $\left(i_{\text {max }}=\arg \max \left(R_{k}^{\prime}\right)\right)$ and minimum $\left(i_{\min }=\arg \min \left(R_{k}^{\prime}\right)\right)$ of $R_{k}^{\prime}$. And update ppi value, $p_{i_{\max }}=p_{i_{\max }}+1, p_{i_{\min }}=p_{i_{\min }}+1$

Step5 In the set of $\left\{p_{i}\right\}_{i=1}^{n}$, if nonzero value $<\mathrm{m}$, then all the pixels with nonzero ppi are endmember, that is $E=\left\{\left\{x_{i}\right\} \mid p_{i}>0, i=1,2, \cdots, n\right\}$, else set the endmember with the top $\mathrm{m}$ ppi.

In this paper, we chose the 15 dimensions of the MNF transform as the effective input data of PPI processing. Through repeated experiments, we set the iterations as 5000 times, the endmember number as three. That was oil, water and cloud. The total number of pixels in the study area was 265 . The above experiment was completed in the ENVI software. PPI was a supervised endmember extraction method here. The extraction results are shown in fig.3.

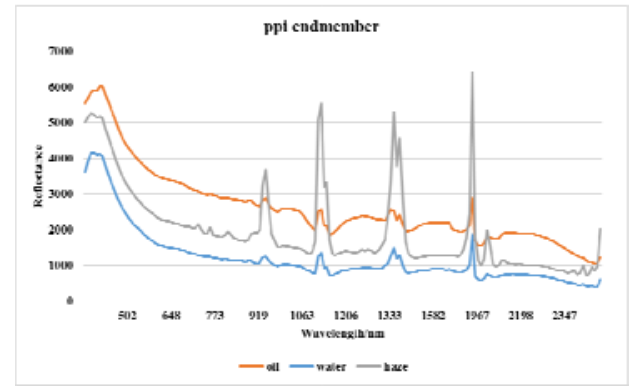

Figure 3. PPI endmember spectra

\section{B. UOSP}

This paper also presented an unsupervised method to extract endmember called UOSP, which overcame the shortcomings of traditional techniques to require a priori spectral knowledge [5].

Set $r$ as the pixel vector of hyperspectral image [6]. Set $S$ as the endmember spectral matrix and $\alpha$ as $\mathrm{m}$ dimension vector. Define $n$ as the random noise. The linear mixing spectral model can be created as in (1).

$$
r=S \alpha+n
$$

And divide $S$ into interested signal $d$ and uninterested $U, S=[d, U]$, then (1) can be written as in (2).

$$
r=d \alpha_{d}+U \alpha_{U}+n
$$

The endmember's position is thought to be at the angular point of an image. Some automatic endmember extraction methods are based on convex polygon algorithm condition. Although it is difficult to find the whole endmember matrix $S$, it is easy to search one of the endmembers in the image.

Set convex set $\Omega$ as the hyperspectral cube, if $\xi_{1}=\arg \max \{\Omega\}$, then $\xi_{1}$ is the angular point of $\Omega$. Else there exists $x \in \Omega, y \in \Omega$, make $\xi_{1}=f_{x}+(1-f) y, f \in(0,1)$. That is, there must be a larger than $\xi_{1}$ between $\mathrm{x}$ and $\mathrm{y}$, which contradicts with $\xi_{1}=\arg \max \{\Omega\}$. So regardless of the noise, in the image cube the maximum vector of the pixel is an endmember vector, which is the brightest one.

When the SNR of the image is low, the problem comes to determine whether $\xi_{1}$ represents noise or endmember. So suppose the endmember in the space is in a certain distribution and accumulation. Set $\xi_{1}$ as $\left(X_{i}, Y_{i}\right)$, window in some size was centering on $\xi_{1}$, and inspect the pixels similar with $\xi_{1}$. If the number is larger than some value $i$, then $\xi_{1}$ is thought to be endmember vector, else to be noise.

By searching for the maximum pixel vector in the image data, we can always find one of the endmember spectral signal $d=\xi_{1}$, and use projection matrix $P_{d}^{\perp}$ to remove the signal $d$ in the image.

$$
P_{d}^{\perp}=I-d\left(d^{T} d\right)^{-1} d^{T}
$$

Once the signal $d$ is known, $P_{d}^{\perp}$ is determined. And $\mathrm{d}$ is always found in the dataset $\Omega$. So the projection matrix can be calculated without using $U$ matrix.

According to (2) and (3),

$$
P_{d}^{\perp} r=P_{d}^{\perp} U \alpha_{U}+P_{d}^{\perp} n
$$

On the right side of (4), there is no more signal $d$, which not only removes $d$ from the original image, but also compresses the noise into $P_{d}^{\perp} n$.

Notice the right side $P_{d}^{\perp} r$ is already known, take $P_{d}^{\perp} r$ as a new convex set $\hat{\Omega}$, then another $\xi_{2}=\arg \max \{\hat{\Omega}\}$ can be found, $\xi_{2}$ is another endmember vector. By now the projection matrix can be calculated as in (5).

$$
P_{\tilde{d}}^{\perp}=I-\tilde{d}\left(\tilde{d}^{T} \tilde{d}\right)^{-1} \tilde{d}^{T}
$$

Where $\tilde{d}=\left[\xi_{1}, \xi_{2}\right]$, and so on, the $\mathrm{j}+1$ times projection matrix $P^{\perp}=I-\hat{d}\left(\hat{d}^{T} \hat{d}\right)^{-i} \hat{d}^{T}, \hat{d}=\left[\xi_{1}, \xi_{2}, \cdots, \xi_{j}\right]$. The end condition is to get the specified number of the endmember, or by the specified RMSE. So that we can get all $\mathrm{K}$ endmember vector, that is, the endmember spectral matrix $\mathrm{S}$.

In this paper, the UOSP method was used to automatically select the endmember spectrum. The window size was selected as $R=15$, the total number of similar pixels in the window was $W=120$, a total of iterations was set to 5 , the decomposition of the error is 0.0008 . The following table is the intermediate result of the iterative calculation. 
TABLE I. ITERATIVE CALCULATION

\begin{tabular}{|c|c|c|c|c|c|}
\hline & $\begin{array}{c}\text { Iteratio } \\
\mathbf{n 1}\end{array}$ & $\begin{array}{c}\text { Iteratio } \\
\mathbf{n} 2\end{array}$ & $\begin{array}{c}\text { Iteratio } \\
\mathbf{n 3}\end{array}$ & $\begin{array}{c}\text { Iteratio } \\
\mathbf{n 4}\end{array}$ & $\begin{array}{c}\text { Iteratio } \\
\mathbf{n 5}\end{array}$ \\
\hline $\begin{array}{c}\text { coordinat } \\
\text { e }\end{array}$ & $(30,18)$ & $(57,3)$ & $(84,108)$ & $\begin{array}{c}(268, \\
126)\end{array}$ & $\begin{array}{c}(129, \\
177)\end{array}$ \\
\hline $\begin{array}{c}\text { Similar } \\
\text { pixels }\end{array}$ & 181 & 159 & 152 & 148 & 167 \\
\hline RMSE & 0.6954 & 0.1866 & 0.0128 & 0.0029 & 0.0008 \\
\hline
\end{tabular}

As is shown in Table I, the relative error of each iteration is reduced sharply, and the spectral curves of three kinds of features are obtained, the UOSP endmember spectra are shown in Fig. 4.

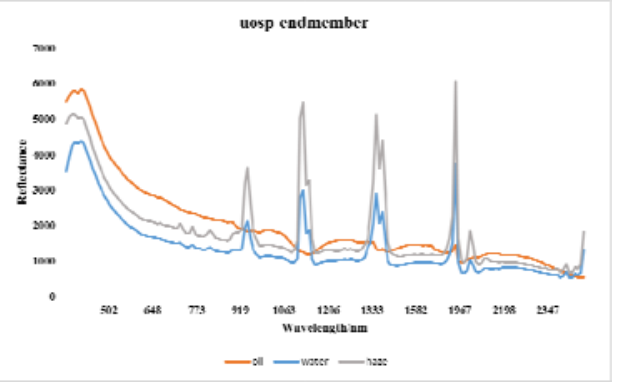

Figure 4. UOSP endmember spectra

\section{Evaluation of Endmember Extraction}

Due to external conditions, such as imaging and time imaging condition is different, the absolute value of measured reference endmember spectra and hyperspectral endmember spectra varied widely. But the basic shape of the curves is similar. At the wavelength of $773 \mathrm{~nm}$ there are both several reflection peaks. So the evaluation method was based on spectral angle. There are similar characteristics among the reference spectra of different thickness oil, so the thickness of $10 \mu \mathrm{m}$ oil film and the maximum thickness of $2000 \mu \mathrm{m}$ were used as the reference spectra. The spectral angle evaluation is shown in the Table II.

TABLE II. SPECTRAL ANGLE OF REFERENCE SPECTRA AND ENDMEMBER SPECTRA EXTRACTED BY PPI AND UOSP

\begin{tabular}{|c|c|c|}
\hline Feature & PPI & UOSP \\
\hline Oill $(10 \mu \mathrm{m})$ & 0.55 & 0.49 \\
\hline Water & 0.42 & 0.45 \\
\hline Oil $(2000 \mu \mathrm{m})$ & 0.36 & 0.28 \\
\hline Water & 0.42 & 0.45 \\
\hline
\end{tabular}

From the table, the spectral angle between reference spectra and UOSP endmember spectra was smaller than that of PPI, so the accuracy is higher. But the result of water extraction was opposite. Overall, the oil spill film thickness in the image was closer to $2000 \mu \mathrm{m}$.

IV.

\section{FCLS ABUNDANCE ESTIMATION}

The existing spectral mixture models mainly include linear and nonlinear models. Linear spectral mixture model is to assume that the spectra is a linear combination of pure independent endmember. Its purpose is to get the endmember proportion contained in the mixed pixels by analysis and calculation, called abundance estimation.

Fully constrained least squares abundance estimation is the combination of sun-to-one constrained least squares and non-negative constrained least squares [7]. The FCLS has the highest accuracy. It could be described as following.

The mixed pixel decomposition could be calculated as in (6).

$$
\alpha_{l s}=\left(S^{T} S\right)^{-1} S^{T} r
$$

The fully constrained condition is $\sum_{i=1}^{k} T_{i}=1$ and $T_{i} \geq 0$. The difficulty is to ensure $T_{i} \geq 0$.

$$
M=\left[\begin{array}{c}
S \\
1^{T}
\end{array}\right], 1^{T}=(1,1, \cdots, 1), \rho=\left[\begin{array}{c}
r \\
1
\end{array}\right], \text { a } k \text {-dimension vector }
$$

$\beta=\left[U_{1}, U_{2}, \cdots, U_{\mathrm{k}}\right], U_{\mathrm{i}}>0$ is introduced, to construct a Lagrange multiplier equations as in (7).

$$
J=\frac{1}{2}(\rho-M \alpha)^{\mathrm{T}}(\rho-M \alpha)+\lambda(\alpha-\beta)
$$

Under the condition of $\alpha=\beta$,

$$
\left.\frac{\partial J}{\partial \alpha}\right|_{c l s}=0 \Rightarrow\left(M^{\mathrm{T}} M\right) \alpha_{c l s}-M^{\mathrm{T}} \rho+\lambda=0
$$

Where cls denotes constrained linear spectral. So the iterative equations are derived

$$
\begin{gathered}
\alpha_{c l s}=\alpha_{l s}-\left(M^{\mathrm{T}} M\right)^{-1} \lambda \\
\lambda=M^{T}\left(\rho-M \alpha_{c l s}\right)
\end{gathered}
$$

As in (9) and (10), $\alpha_{c l s}$ and $\lambda$ could be calculated.

Two methods of endmember extraction abundance map are shown in Fig.5 and Fig.6. The pixel value represents the endmember proportion of the pixel. The higher the level of gray value, the higher accuracy estimation. At last the root mean square error method was used to evaluate the abundance results.

The oil abundance by UOSP was larger than that by PPI. And in the middle of the image, there was a piece of cloud where the pixels were with low gray value estimated correctly by UOSP. Since large area of the bottom was oil, the water abundance in the bottom of the image was also correct by UOSP. In the abundance estimation of haze, the UOSP method was wrong to regard some oil as haze in the bottom right corner. The RMSE also showed the UOSP-FCLS was better than PPIFCLS. The RMSE of UOSP was 106.54 relative to 148.42 of

PPI. 


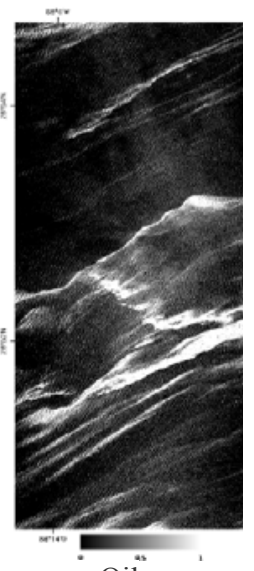

a. Oil

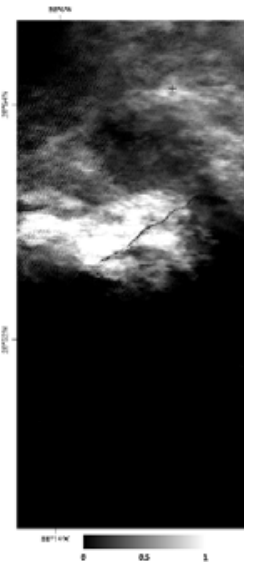

c. Haze

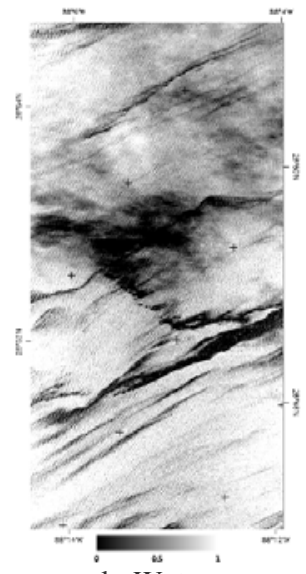

b. Water

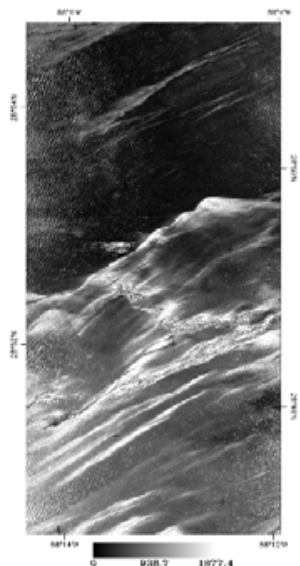

d. RMSE
Figure 5. PPI-FCLS Abundance Estimation

\section{SUMMARY}

This paper compared oil and water endmember extraction method of PPI and UOSP to the measured reference spectra. Endmember evaluation is an important task for analysis of hyperspectral unmixing. Through the unsupervised OSP endmember extraction, the abundance estimation was more reliable than the supervised PPI endmember extraction.

\section{ACKNOWLEDGMENT}

This work was supported in part by the Natural Science Foundation of China under Grant No. 41171329, the Special Scientific Research Project of Oceanic Public Welfare Profession of China by State Oceanic Administration under Grant No. 201305002, and the Fundamental Research Funds for the Central Universities No. 3132016003.

\section{REFERENCES}

[1] P Sidike,J Khan,M Alam,S Bhuiyan, "Spectral unmixing of hyperspectral data for oil spill detection." Proceedings of SPIE -
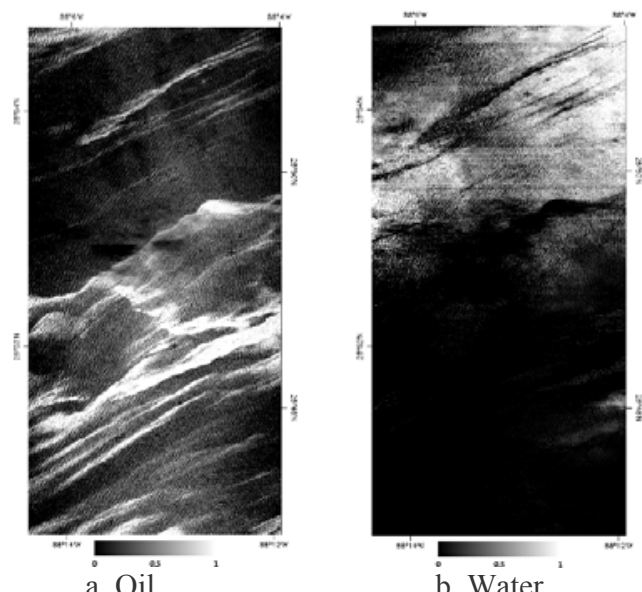

b. Water

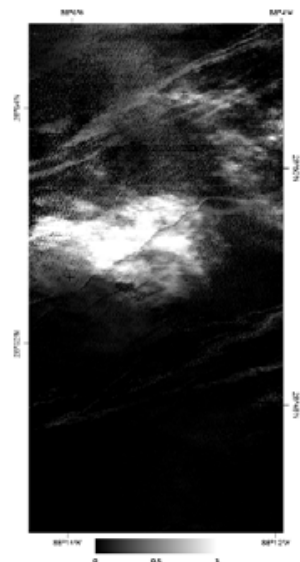

c. Haze

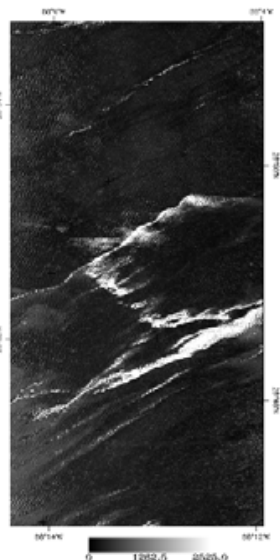

d. RMSE

Figure 6. UOSP-FCLS Abundance Estimation

The International Society for Optical Engineering, vol. 8498, pp 84981B-84981B-10, 2012,.

[2] D. Sykas, V. Karathanassi, C Andreou, P Kolokoussis, "Oil spill thickness estimation using unmixing methods," 2011 3rd Workshop on Hyperspectral Image and Signal Processing: Evolution in Remote Sensing (WHISPERS), IEEE. Lisbon, Portugal, pp.1-4, June 2011.

[3] B Liu, Y Li, L Han, "Identification of Oil Type Using Spectral Reflectance Characteristics," Spectroscopy and Spectral Analysis, vol.36, no.4, pp.1100-1103, 2016.

[4] DM Rogge,B Rivard,J Zhang,A Sanchez,J Harris,J Feng, "Integration of spatial-spectral information for the improved extraction of endmembers," REMOTE SENS ENVIRON, vol.110, no.33, pp.287-303, 2007.

[5] C M Wang, C C Chen, S C Yang, P C Chung, Y N Chung, "Unsupervised orthogonal subspace projection approach to magnetic resonance image classification," OPT ENG, vol.41, no.7, pp.1546-1557, 2002.

[6] H Ren, C I Chang, "A generalized orthogonal subspace projection approach to unsupervised multispectral image classification," IEEE Trans. Geosci. Remote Sensing, vol.38, no.6, pp.2515-2528, 2000.

[7] D C Heinz, "Fully constrained least squares linear spectral mixture analysis method for material quantification in hyperspectral imagery," IEEE Trans. Geosci. Remote Sensing, vol.39, no.3, pp.529-545, 2001 . 